\title{
Gestión ambiental en las empresas mixtas de un complejo petroquímico de Venezuela
}

\author{
Environmental management in mixed companies of a Venezuela \\ petrochemical complex
}

Lorena del Carmen Fuentes Spooner

Ifuentesp89@gmail.com

Código ORCID: 0000-0002-9037-4692

Universidad del Zulia. Maracaibo, Venezuela
María Alejandra Güere Nava

magn2507@gmail.com

Código ORCID: 0000-0003-4579-8119

Universidad del Zulia. Maracaibo, Venezuela

Yajaira Alvarado

yaalv26@gmail.com

Código ORCID: 0000-0001-5221-2302

Universidad del Zulia. Maracaibo, Venezuela

Articulo recibido en julio 2019 Arbitrado en agosto 2019 Publicado en enero 2020

\section{Resumen}

La presente investigación tuvo como objetivo analizar la gestión ambiental en las empresas mixtas del complejo petroquímico Ana María Campos del estado Zulia-Venezuela. El estudio fue descriptivo, de campo, univariable y transeccional. La población estuvo conformada por tres empresas con un total de 35 informantes. Se aplicó un cuestionario con cinco alternativas de respuesta, validado mediante el juicio de cinco expertos y con un alto coeficiente de confiabilidad (Alfa de Cronbach=0,94). El análisis de los datos se realizó a través de la media aritmética. Los resultados muestran que las empresas siempre implementan mecanismos para controlar los aspectos ambientales derivados de sus actividades productivas, pero casi nunca llevan a cabo el proceso de gestión ambiental. Se concluye que las empresas objeto de estudio cumplen con la regulación ambiental vigente en cuanto a la ejecución de los estudios ambientales, pero deben elaborar e implementar un sistema de gestión ambiental.

Palabras clave: Aspectos ambientales, empresas petroquímicas, gestión ambiental, proceso de gestión ambiental

Abstract

The objective of the present investigation was to analyze the environmental management in the mixed companies of the Ana María Campos petrochemical complex of the ZuliaVenezuela state. The study was descriptive, field, univariate and transectional. The population was made up of three companies with a total of 35 informants. A questionnaire with five response alternatives was applied, validated by the judgment of five experts and with a high reliability coefficient (Cronbach's Alpha $=0.94$ ). Data analysis was performed using the arithmetic mean. The results show that companies always implement mechanisms to control the environmental aspects derived from their productive activities, but almost never carry out the environmental management process. It is concluded that the companies under study comply with the environmental regulations in force regarding the execution of environmental studies, but must prepare and implement an environmental management system.

Keywords: Environmental aspects, petrochemical companies, environmental management, environmental management process 


\section{INTRODUCCIÓN}

A nivel mundial se ha comenzado a difundir la conciencia ambiental como un tema primordial para el buen desarrollo del planeta, ya que éste se encuentra sometido a grandes presiones debido al deterioro por el uso indiscriminado de los recursos naturales renovables y no renovables, al inadecuado consumo de energía, y al incorrecto manejo de los residuos y desechos generados por la sociedad. Lo anteriormente expuesto ha traído como consecuencia un desequilibrio en la atmósfera, causado por la producción antrópica de gases de efecto invernadero, lo cual ha empezado a inducir severos cambios en el clima.

Desde la revolución del siglo XVIII la industria ha ido en constante desarrollo, construyendo su índice de crecimiento en parámetros de la calidad de vida de su población bajo el enfoque del desarrollo económico. Debido a la preocupación de los países industrializados por el daño ambiental, el cual es provocado por la contaminación gradual en el planeta, desde la década de los años 70 del siglo XX se aplica el concepto de gestión ambiental.

En este orden de ideas, para Pousa (2006:2), la gestión ambiental se define "como el conjunto de acciones encaminadas a lograr la máxima racionalidad en el proceso de decisión relativo a la conservación, defensa, protección y mejora del medio ambiente, basándose en una coordinada relación ciudadana multidisciplinaria".

Por su parte, en la Ley Orgánica del Ambiente (2006:4) se define la gestión ambiental como "todas las actividades de la función administrativa, que determinen $y$ desarrollen las políticas, objetivos $y$ responsabilidades ambientales y su implementación, a través de la planificación, el control, la conservación y el mejoramiento del medio ambiente".

Es por eso que, las empresas deben tener en cuenta a la hora de planificar sus actividades, bien sea en el corto, mediano y largo plazo, un factor muy importante como es el ambiente, el cual debe ser integrado en la gestión de la empresa. Dicha integración se consigue con la implantación de un sistema de gestión ambiental certificado ante un ente reconocido internacionalmente para así garantizar que sus actividades se están desarrollando en armonía con el ambiente.

En este sentido, las exigencias legales y reglamentarias han generado que las empresas tengan una creciente concienciación en el tema ambiental, al asumir que la protección y proyección al medio ambiente es una exigencia, la cual adquiere gran relevancia para la supervivencia de las empresas.

En consecuencia, éstas deben concentrarse cada vez más en medidas preventivas; es decir, las empresas deben establecer un sistema de gestión ambiental que les permita estar en conformidad con todas las disposiciones vigentes en cuanto a normativas ambientales.

Dentro de este marco, para Pousa (2006:3) un sistema de gestión ambiental se define como

"un instrumento o herramienta que posee la empresa como ayuda en las actividades de su gestión medio ambiental aportando la base para orientar, encauzar, medir y evaluar su funcionamiento con el fin de asegurar que sus operaciones se lleven a cabo de una manera consecuente con la reglamentación aplicable y con la política corporativa". 
Adicionalmente, Herrero y Sáez (2014:110) consideran que un sistema de gestión ambiental "es la parte de un sistema de gestión de una organización, empleada para desarrollar e implementar su política ambiental y gestionar sus aspectos ambientales".

En síntesis, un sistema de gestión ambiental involucra la planificación, implantación, revisión, mejora de los procedimientos y acciones que permiten a una organización realizar sus actividades en armonía con el ambiente y así garantizar el cumplimiento de sus objetivos y metas ambientales.

Cabe destacar la existencia de normas internacionales estandarizadas provenientes de la familia ISO 14000 (International Organization for Standardization - ISO). Ésta en 1993 emprendió la tarea de estandarizar la gestión ambiental basándose en la norma británica BS7750, que fue publicada oficialmente por la British Standards Institution (BSI) previa a la Reunión Mundial de la ONU sobre el ambiente en 1992. Estas normas han sido creadas para proporcionar información sobre lo que se espera que las empresas cumplan para controlar sus actividades productivas, considerando sus impactos negativos.

Así mismo, la Norma Internacional ISO 14001 (2015) es una norma que rige los lineamientos y requisitos a seguir para la elaboración de un sistema de gestión ambiental para las empresas. La misma ha sido adoptada por varias empresas a nivel internacional, para controlar los posibles impactos negativos de sus actividades sobre el medio ambiente. Al respecto, en la Norma Internacional ISO 140001 (2015:vii) se afirma lo que a continuación se expone:
"El propósito de esta Norma Internacional es proporcionar a las organizaciones un marco de referencia para proteger el medio ambiente y responder a las condiciones ambientales cambiantes, en equilibrio con las necesidades socioeconómicas. Esta norma específica requisitos que permitan que una organización logre los resultados previstos que ha establecido para su sistema de gestión ambiental".

Dicho en otras palabras, la Norma Internacional ISO 14.001 (2015) permite proporcionar a las empresas un Sistema de Gestión Ambiental (SGA) efectivo, contrastado e integrado en el resto de las actividades productivas. La profusión de normativas y leyes ambientales hace que en el desarrollo cotidiano de la actividad empresarial surja la necesidad de solventar aspectos o condicionantes ambientales. La misma describe el proceso a seguir por las empresas y le exige respetar las leyes ambientales nacionales. Además, esta norma tiene "orientación hacia los modelos de excelencia, facilitando su integración con los métodos de mejora y competitividad" (Cuadros y Téllez, 2019:154), de manera que se puedan crear sistemas integrados en los cuales se incluyan otras normativas para lograr un desarrollo sostenible y así optimizar el desempeño en las funciones ambientales, económicas y sociales de las organizaciones.

En el caso de Venezuela, aunque existe un organismo gubernamental encargado de la protección del medio ambiente, como lo es el Ministerio del Poder Popular para el 
Ecosocialismo, antiguo Ministerio del Ambiente, los autores Infante y Robles (2008) señalan que la mayoría de las empresas públicas venezolanas no cuentan con un sistema de gestión ambiental certificado bajo la Norma Internacional ISO 14001 (2015).

Por otra parte, es importante acotar que en los últimos años el estado venezolano, a través de la Constitución de la República Bolivariana de Venezuela, leyes, normas y decretos en el área ambiental, ha hecho hincapié en las empresas, bien sea públicas o privadas, para que cumplan con lo estipulado al respecto, generando de esta manera un interés por producir de forma ambientalmente segura, acoplándose a las normativas ambientales vigentes.

En el caso específico de la Industria Petroquímica de Venezuela (Pequiven), creada en el año 1977 y asumiendo las operaciones del Instituto Venezolano de Petroquímica (IVP), fundado en 1955; ésta ha pasado por distintas etapas de reestructuración, consolidación y expansión, con lo que se ha logrado ampliar su campo de operaciones, desarrollando un importante mercado interno y externo para sus productos. En 1968 inician las operaciones del complejo Petroquímico Morón (Carabobo) y Ana María Campos conocido como "El Tablazo" y finalmente en el año 1986 lo hace el Complejo Antonio José de Anzoátegui, ubicado al este de Barcelona, uno de los más grandes establecimientos petroquímicos de Latinoamérica.

La construcción de la industria petroquímica aumentó significativamente la expansión de las actividades petroquímicas venezolanas e impulsó el aprovechamiento del gas natural como fuente básica de insumos para estas operaciones. Hoy en día la Petroquímica de Venezuela es una corporación independiente, adscrita al Ministerio del Poder Popular para la Energía y Petróleo.

El complejo petroquímico Ana María Campos, objeto de estudio en esta investigación, se caracteriza por poseer las actividades más complejas y dinámicas a escala mundial. Se identifica principalmente por un rápido y continuo desarrollo que la obliga a utilizar tecnologías altamente especializadas, intensivos requerimientos de capital y recursos humanos calificados desde el punto de vista gerencial y técnico.

Para coadyuvar con el desarrollo del sector petroquímico nacional, se promovió la incorporación de empresas privadas nacionales y extranjeras, para formar, junto a la Petroquímica del Estado, específicamente en el Complejo Petroquímico Ana María Campos, las denominadas empresas mixtas. Dichas empresas tienen como responsabilidad la producción, almacenaje, transporte, distribución y comercialización de las olefinas y las resinas plásticas, a nivel nacional e internacional. Si bien es cierto que el complejo petroquímico Ana María Campos es parte de la base fundamental en la economía del país, sus actividades generan impactos negativos al ambiente que al no ser controladas pueden ocasionar daños irreversibles al mismo. Ello amerita la existencia de un adecuado control ambiental para evitar, en lo posible, que sus operaciones ocasionen perjuicios al entorno.

No obstante, debido a la observación directa en las empresas mixtas del complejo petroquímico Ana María Campos, se detectó que no todas cuentan con una política 
ambiental bien definida en concordancia a las necesidades ambientales de la organización, además el personal encargado de supervisar y llevar a cabo la gestión ambiental, posiblemente no cuenta con la formación necesaria ni los recursos tanto técnicos como financieros para cumplir cabalmente con los lineamientos $y$ procedimientos ambientales estipulados en la normativa regulatoria para tal fin.

De acuerdo a lo anteriormente expuesto, la presente investigación tiene como objetivo analizar la gestión ambiental en las empresas mixtas del complejo petroquímico Ana María Campos del estado Zulia, para de esta manera determinar si las mismas cuentan con un sistema de gestión ambiental bien definido, el cual en un futuro pueda ser auditado y posteriormente certificado como aval de una correcta gestión ambiental en los procesos que se desempeñan en cada planta.

\section{MÉTODO}

La presente investigación estuvo enmarcada en el nivel descriptivo (Hurtado, 2010). El diseño para la presente investigación, se clasificó como no experimental, de campo, univariable, contemporáneo y transeccional. La población estuvo conformada por tres empresas (PRALCA, PROPILVEN y POLINTER) del Complejo Petroquímico "Ana María Campos" del estado Zulia, con un total de treinta y cinco sujetos informantes, entre los cuales figuraron representantes de la dirección, líderes del proceso de gestión de calidad, superintendentes de calidad, analistas de calidad y auditores. Esta población se distribuyó tal y como se especifica en la Tabla 1.

Tabla 1. Distribución de la población

\begin{tabular}{clc}
\hline Empresa & \multicolumn{1}{c}{ Cargos que desempeñan } & $N^{\circ}$ de sujetos \\
\hline \multirow{3}{*}{ PROPILVEN } & 1 \\
& Coordinador SHA & 3 \\
& Ingeniero SHA & 1 \\
& Inspector SHA & 1 \\
& Representante a la dirección & 2 \\
& Analista de la Calidad & 1 \\
& Auditor & 1 \\
& Coordinador SHA & 4 \\
& Líder Control Ambiental y Saneamiento & 1 \\
POLINTER & Líder del proceso Sistema Gestión de la Calidad & 6 \\
& Analista de la Calidad & 3 \\
& Auditor & 1 \\
& Coordinador SHA & 2 \\
& Ingeniero SHA & 2 \\
& Inspector SHA & 1 \\
& Superintendente de calidad & 3 \\
& Analista de la Calidad & 2 \\
& Auditor & 35
\end{tabular}


Se aplicó la técnica de la encuesta y se empleó un cuestionario con cinco categorías de respuesta: siempre, casi siempre, a veces, casi nunca y nunca. La validez del instrumento se estableció a través del juicio de cinco expertos y la confiabilidad mediante el coeficiente Alfa de Cronbach, cuyo valor $(0,94)$ permitió establecer una muy alta confiabilidad. El análisis de los datos se realizó a través de la media aritmética. En este sentido se elaboró un baremo con diversas alternativas de respuesta para el análisis de los valores promedio, cuyo fin es medir el grado de presencia de la variable gestión ambiental (Tabla 2).

Tabla 2. Baremo para el análisis de la media aritmética

\begin{tabular}{|c|c|c|c|c|}
\hline \multicolumn{2}{|c|}{ Alternativas } & Categorías & Intervalos & Descripción \\
\hline Siempre & $\mathrm{S}$ & $\begin{array}{l}\text { Muy alta } \\
\text { presencia }\end{array}$ & $4,21<5,00$ & $\begin{array}{l}\text { El elemento analizado se encuentra dentro } \\
\text { de una muy alta frecuencia para las } \\
\text { dimensiones e indicadores planteados. }\end{array}$ \\
\hline Casi Siempre & CS & Alta presencia & $3,41<4,2$ & $\begin{array}{l}\text { El elemento analizado se encuentra dentro } \\
\text { de una alta frecuencia para las dimensiones } \\
\text { e indicadores planteados. }\end{array}$ \\
\hline A veces & $\mathrm{AV}$ & $\begin{array}{l}\text { Moderada } \\
\text { presencia }\end{array}$ & $2,61<3,4$ & $\begin{array}{l}\text { El elemento analizado se encuentra dentro } \\
\text { de una moderada frecuencia para las } \\
\text { dimensiones e indicadores planteados. }\end{array}$ \\
\hline Casi Nunca & $\mathrm{CN}$ & Baja presencia & $1,81<2,6$ & $\begin{array}{l}\text { El elemento analizado se encuentra dentro } \\
\text { de una baja frecuencia para las dimensiones } \\
\text { e indicadores planteados. }\end{array}$ \\
\hline Nunca & $\mathrm{N}$ & $\begin{array}{l}\text { Muy baja } \\
\text { presencia }\end{array}$ & $1,00<1,8$ & $\begin{array}{l}\text { El elemento analizado se encuentra dentro } \\
\text { de una muy baja frecuencia para las } \\
\text { dimensiones e indicadores planteados. }\end{array}$ \\
\hline
\end{tabular}

A efectos de esta investigación el análisis se realizó mediante el paquete estadístico de Microsoft Excel con el cual se procesaron los datos para obtener la medida de tendencia central (media aritmética) de acuerdo al instrumento diseñado, a fin de obtener el puntaje que representa la gestión ambiental en las empresas mixtas del complejo Petroquímico Ana María Campos del estado Zulia, tanto en las dimensiones (aspectos ambientales y proceso de gestión ambiental) como en sus indicadores.

\section{RESULTADOS}

El análisis de la media aritmética en las empresas mixtas POLINTER, PROPILVEN y PRALCA del complejo Petroquímico Ana María Campos del estado Zulia (Venezuela) con la participación de 35 sujetos informantes, refleja los resultados para las dimensiones aspectos ambientales y proceso de gestión ambiental. 


\section{Aspectos ambientales}

Los resultados en cuanto a los aspectos ambientales para las empresas objeto de estudio se representan en la Figura 1 a través de los indicadores: generación de efluentes, generación de residuos sólidos no peligrosos, generación de desechos peligrosos, emisiones atmosféricas y emisiones de ruido.

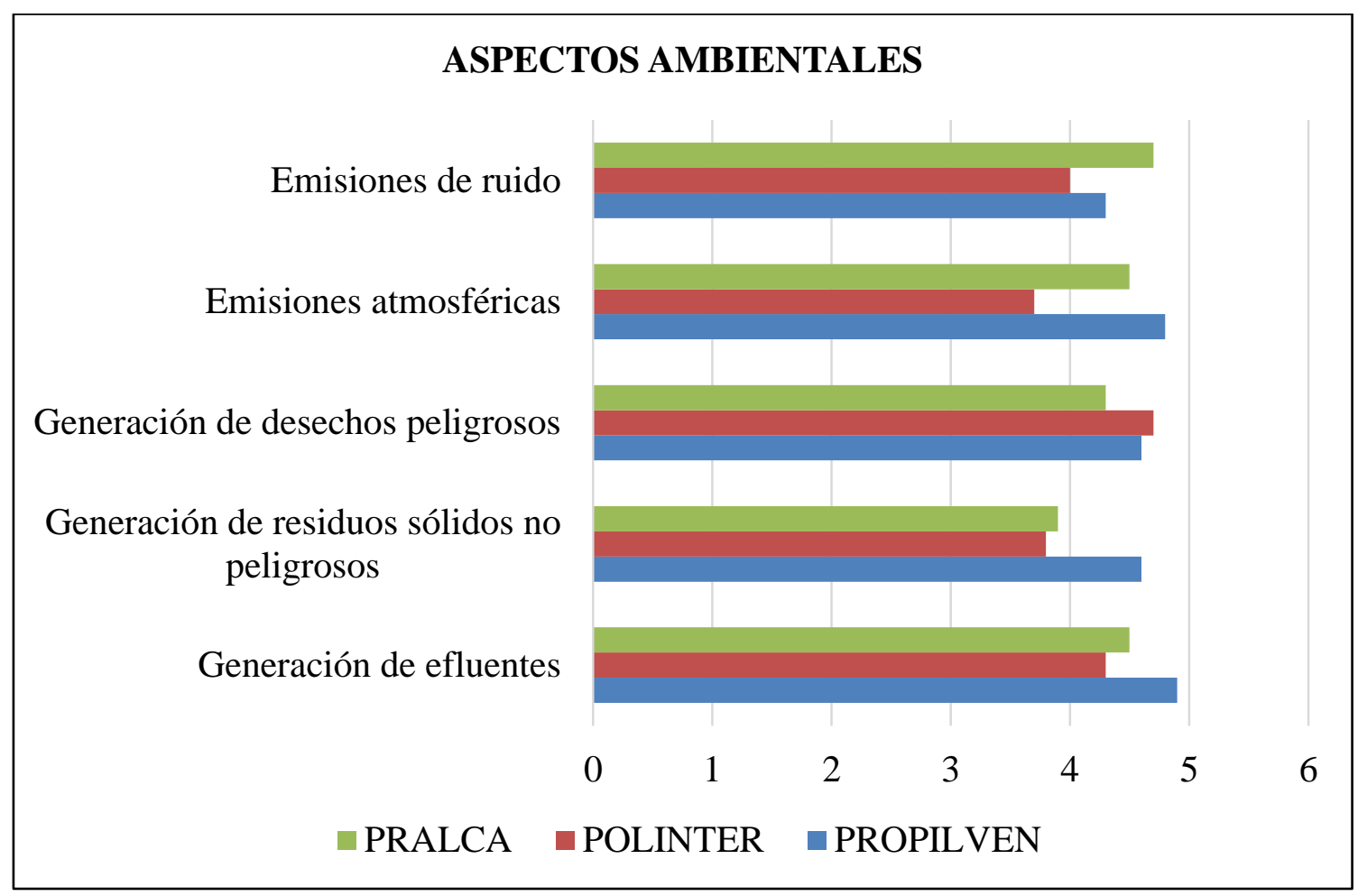

Figura 1. Comportamiento de los indicadores correspondientes a la dimensión aspectos ambientales en las empresas mixtas del complejo Petroquímico Ana María Campos.

\section{Generación de efluentes}

El indicador generación de efluentes se sitúa en la categoría muy alta presencia, pues el índice obtenido es de 4,6. En el gráfico 1 se observa que la empresa con mayor aplicación de medidas para controlar el impacto negativo que pudiera causar la generación de efluentes es PROPILVEN, la cual arroja una media de 4,9 que se ubica en la categoría muy alta presencia, seguida de POLINTER y de PRALCA con promedios de 4,3 y 4,5 , respectivamente; que igualmente corresponden a la categoría muy alta presencia.
El hecho de que los resultados del indicador generación de efluentes refleje un valor de 4,6 como promedio, indica que las empresas objeto de estudio siempre realizan la caracterización de sus efluentes y para ello los clasifican e identifican como orgánicos e inorgánicos; así mismo, se detecta que las empresas mencionadas poseen un sistema de pretratamiento que permite adecuar sus efluentes antes de ser vertidos al Lago de Maracaibo para evitar su contaminación.

Lo anterior confirma lo señalado por Castells (2012), pues al tratarse adecuadamente los efluentes generados, se 
evita la contaminación de los lagos, puesto que se garantiza la eliminación de los contaminantes en las aguas derivadas del proceso productivo.

\section{Generación de residuos sólidos no peligrosos}

Con relación al indicador generación de residuos sólidos no peligrosos, se observa que los resultados obtenidos se ubican en la categoría alta presencia, pues el índice obtenido es 4,1 . En el gráfico 1 se muestra que la empresa con mayor aplicación de medidas para controlar el impacto negativo que pudiera causar el aspecto generación de residuos sólidos no peligrosos es PROPILVEN, la cual arroja un valor de 4,6 como promedio, vinculado a la categoría muy alta presencia; en segundo lugar se encuentra la empresa PRALCA con una media aritmética correspondiente a 3,9 y en último lugar está POLINTER con un valor de 3,8 como promedio, asociados a la categoría alta presencia.

En este sentido, se confirma lo señalado por Jiménez y Ortega (2011), ya que es necesario promover la cultura ambiental en las organizaciones, puesto que es vital el compromiso de los empleados con el ambiente para que realicen el manejo adecuado de los residuos no peligrosos generados durante sus actividades.

\section{Generación de desechos peligrosos}

El indicador generación de desechos peligrosos se ubica en la categoría muy alta presencia, cuyo valor alcanza un índice de 4,5. En el gráfico 1 se muestra que la empresa con mayor aplicación de medidas para controlar el impacto negativo que pudiera causar la generación de este tipo de desechos es POLINTER con un promedio de
4,7; seguida de PROPILVEN con una media de 4,6 y finalmente PRALCA que arroja un promedio de 4,3; ubicándose todas en la categoría muy alta presencia.

Los resultados obtenidos muestran una tendencia conforme a la cual las empresas objeto de estudio siempre clasifican los desechos peligrosos para facilitar la disposición adecuada de los mismos, mediante la contratación de una empresa registrada ante el Ministerio del Poder Popular para el Ecosocialismo. Así mismo, las empresas en cuestión siempre consignan ante la dirección estadal ambiental el balance anual de sus desechos peligrosos.

En este orden de ideas, a pesar de los altos costos que implica la contratación de empresas que realicen la disposición adecuada de los desechos peligrosos, tal como lo expresan González y Morales (2010), las empresas objeto de estudio realizan dicha contratación para garantizar la disposición y tratamiento adecuado de este tipo de desechos generados y cumplir con las regulaciones ambientales vigentes.

\section{Emisiones atmosféricas}

Atinente al indicador emisiones atmosféricas, éste se ubica en la categoría correspondiente a muy alta presencia, puesto que se obtuvo un promedio de 4,3. En el gráfico 1 se muestra claramente que la empresa con mayor valor de su media aritmética es PROPILVEN, la cual arroja un índice de 4,8 y se ubica en la categoría referida a muy alta presencia, seguido de PRALCA con un promedio de 4,5 que la sitúa igualmente en la categoría concerniente a muy alta presencia; y finalmente POLINTER con un promedio de 3,7 relacionado con la categoría alta presencia. De acuerdo a lo anteriormente descrito, se supone que las 
empresas en cuestión ejecutan medidas de control para evitar que las emisiones que se derivan de sus actividades ocasionen contaminación del aire.

\section{Emisiones de ruido}

El indicador emisiones de ruido, se ubica en la categoría concerniente a muy alta presencia, cuyo resultado es 4,4. El gráfico 1 refleja que la empresa con mayor promedio obtenido fue PRALCA con un valor de 4,7 asociado a la categoría atinente a muy alta presencia, seguido de PROPILVEN con un promedio de 4,3 que la ubica en la categoría muy alta presencia y por último PRALCA con un promedio de 4,0 vinculado a la categoría alta presencia.

Los resultados obtenidos muestran que en las empresas encuestadas siempre realizan los estudios de ruido ambiental, a través de los cuales se puede evidenciar el cumplimiento del Decreto 2217 (1992), puesto que los niveles de ruido resultantes del estudio se encuentran dentro de los límites máximos permisibles estipulados en el mencionado Decreto.

En síntesis, para la dimensión aspectos ambientales se obtuvo un promedio de 4,4 (Figura 2), lo cual permite afirmar que las empresas objeto de estudio en gran medida implementan mecanismos para controlar los aspectos ambientales derivados de sus actividades productivas asociados a generación de efluentes, generación de residuos sólidos no peligrosos, generación de desechos peligrosos, emisiones atmosféricas y emisiones de ruido. De esta manera mitigan los impactos negativos que ocasionan al ambiente, puesto que siempre cumplen con las regulaciones ambientales vigentes.

En este sentido, los planteamientos anteriores concuerdan con lo señalado por Almeida (2010), pues las empresas objeto de estudio aplican mecanismos de control para minimizar y mitigar los riesgos asociados a los aspectos ambientales derivados de sus actividades productivas.

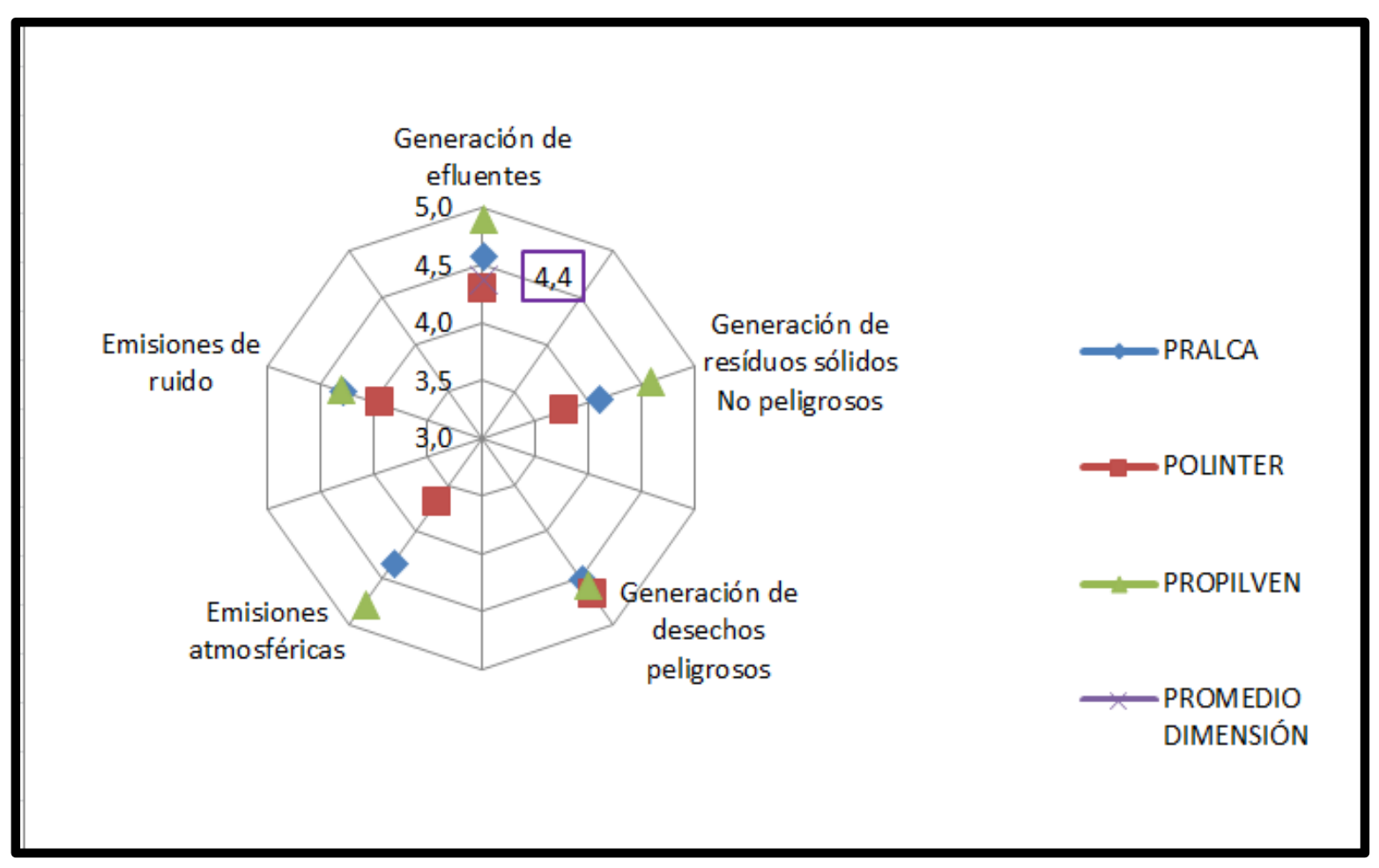

Figura 2. Comportamiento de la dimensión aspectos ambientales en las empresas mixtas del complejo Petroquímico Ana María Campos. 


\section{Proceso de gestión ambiental}

Los resultados en cuanto al proceso de gestión ambiental para las empresas objeto de estudio se representan en el Figura 3 a través de los indicadores: política ambiental, planificación, implementación, verificación y revisión por la dirección.

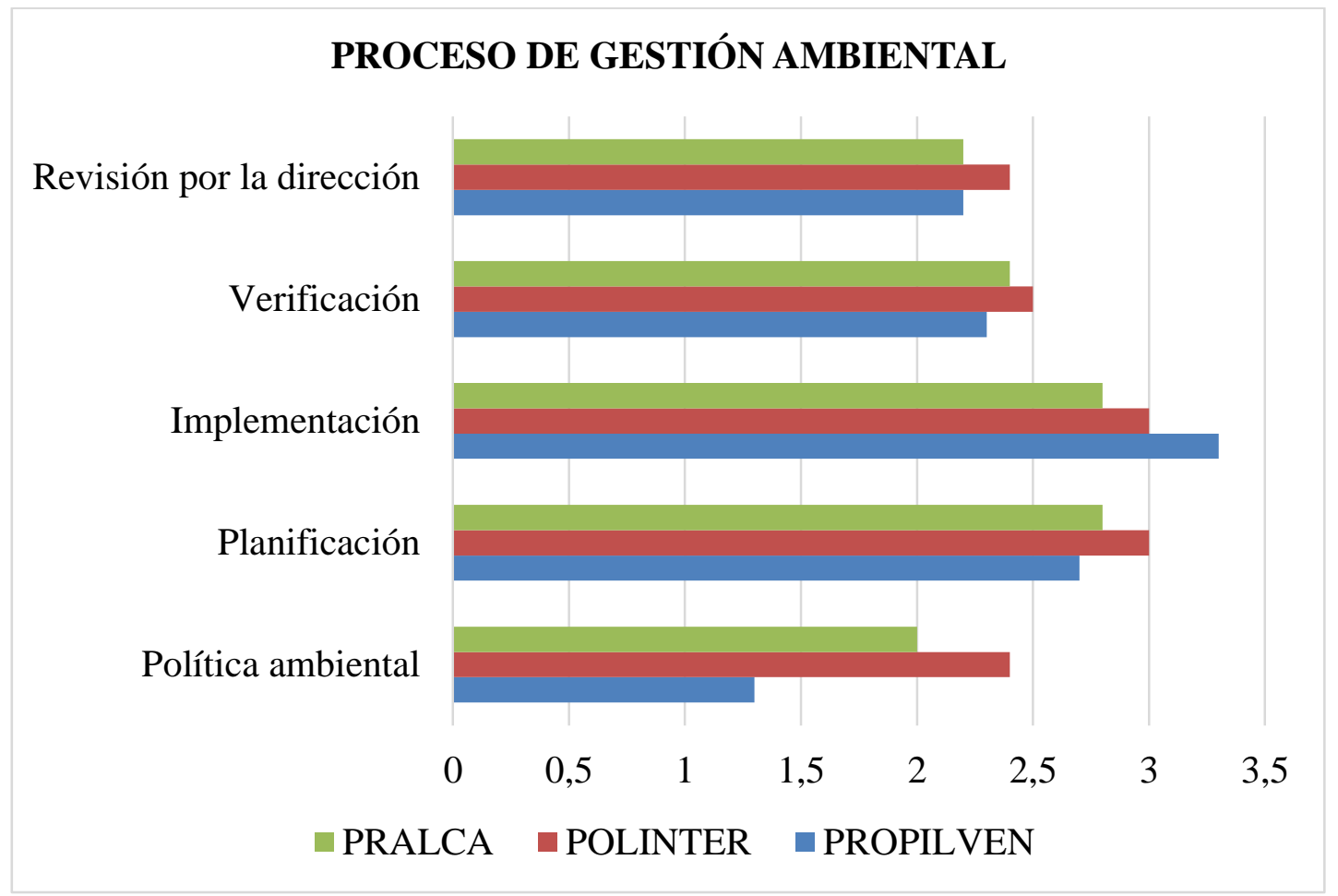

Figura 3. Comportamiento de los indicadores correspondientes a la dimensión proceso de gestión ambiental en las empresas mixtas del complejo Petroquímico Ana María Campos.

\section{Política ambiental}

De acuerdo a las apreciaciones emitidas por el personal que labora en las empresas objeto de estudio, el indicador sobre política ambiental se ubica, según el baremo de análisis, en la categoría baja presencia, dado que el índice obtenido muestra un promedio de 1,9.

En el gráfico 3 se observan los resultados, detectándose claramente que la empresa con una mayor aplicación de la política ambiental es POLINTER, la cual refleja un valor de 2,4 como promedio, seguida de PRALCA con una media de 2,0 y finalmente de PROPILVEN con un promedio de 1,3; valores concernientes a la categoría baja presencia, según el baremo de interpretación.

Los resultados obtenidos revelan que las empresas en cuestión casi nunca definen la política ambiental con la participación activa de los trabajadores, no realizan la divulgación de la misma ni consideran la prevención de la contaminación para definirla. En función de los resultados obtenidos podría interpretarse que las empresas estudiadas no tienen una política ambiental bien definida.

Tomando como fundamento lo señalado por Granero y Ferrando (2007), lo anteriormente expuesto permite inferir que las empresas objeto de estudio carecen de 
una estructura para la acción y el establecimiento, tanto de los objetivos como de las metas ambientales, debido a que no cuentan con una política ambiental bien definida, la cual es considerada, según los autores mencionados, como parte de la estructura del sistema de gestión ambiental.

En este sentido, los resultados difieren de lo señalado por Hernández (2012), pues en las empresas objeto de estudio, la política ambiental representa una debilidad, porque no se encuentra elaborada, esta a su vez debe ser realizada por la alta dirección en conjunto con los empleados de las mismas, dado que son los que poseen los conocimientos acerca de los procesos productivos existentes en cada una de las empresas estudiadas.

\section{Planificación}

Con relación al indicador planificación, en el gráfico 3 se muestran los resultados obtenidos, los cuales se ubican conforme al baremo de interpretación en la categoría inherente a moderada presencia, dado que el promedio obtenido fue de 2,8. En cuanto al comportamiento del indicador planificación en las empresas estudiadas, la empresa POLINTER registra un mayor valor, arrojando un promedio de 3,0; seguido de PRALCA con una media de 2,8 y por último se encuentra PROPILVEN con un promedio de 2,7. Todas las empresas, de acuerdo al baremo de interpretación, se sitúan en la categoría relativa a moderada presencia,.

En función de lo señalado, los resultados demuestran que en las empresas objeto de estudio a veces planifican sus tareas en materia ambiental, las cuales abarcan desde la elaboración del manual de gestión ambiental, definiendo los procedimientos referentes a la identificación de aspectos e impactos ambientales, los requisitos legales aplicables, el establecimiento de los objetivos y las metas ambientales hasta la identificación de las necesidades de formación para posteriormente elaborar el plan de formación ambiental.

Lo anteriormente expuesto se contrapone a los basamentos teóricos expuestos por Matilla (2008), al aseverar que la planificación permite una mayor eficacia en las actividades, pues con ella se evita la improvisación. Por lo tanto, los resultados obtenidos apuntan a que las empresas encuestadas a veces improvisan sus actividades en materia ambiental, ocasionando un mayor consumo de los recursos asignados e incrementando la dificultad para lograr los objetivos y metas ambientales.

Consecuentemente, se supone que no se determinan completamente las acciones previas que deben realizarse para el cumplimiento de la primera fase del ciclo Shewhart/Deming citado por Deming (1989) referida a planificar, la cual es considerada la base en el proceso de gestión ambiental.

Basado en los resultados de este indicador, las empresas objeto de estudio tampoco cumplen con el requisito de la Norma Internacional ISO 14001 (2015) asociado a la planificación bajo el enfoque del ciclo Shewhart/Deming, confirmando lo planteado por Almeida (2010).

\section{Implementación}

Los resultados obtenidos en la medición del indicador implementación se muestran en el gráfico 3, el cual refleja un valor correspondiente a 3,0 como promedio, ubicándolo en la categoría moderada presencia, según el baremo de interpretación. 
Se aprecia claramente que la empresa con mayor promedio obtenido es PRALCA, debido a que presenta un valor correspondiente a 3,4 ; seguido de PROPILVEN con un promedio de 3,3 y por último se encuentra POLINTER con una media aritmética de 2,7. Los valores permiten ubicar a las empresas en la categoría moderada presencia, según el baremo de interpretación.

En este sentido, los resultados permiten visualizar en el gráfico 3 que las empresas objeto de estudio a veces implementan los mecanismos necesarios para ejecutar las actividades planificadas. Concerniente a este aspecto, los encuestados apuntaron que la alta dirección no siente el compromiso necesario para implementar el proceso de gestión ambiental. Por lo tanto, se supone que la asignación de los recursos la realizan medianamente para cumplir solo con los requisitos legales exigidos por las regulaciones ambientales vigentes, dejando atrás la importancia de cumplir con el objetivo principal de la gestión ambiental que es la descarga de cero contaminantes, tal como lo expresan González y Morales (2010).

Los resultados difieren de lo señalado por Acuña et al. (2017) en su estudio con empresas manufactureras de Barranquilla, pues para sus empresarios el principal pilar de la gestión ambiental es la minimización de los residuos generados por los procesos.

Bajo estas premisas, es importante resaltar que la implementación, según la Norma Internacional ISO 14001 (2015), está estrechamente vinculada con la fase hacer, la cual según Álvarez (2013) consiste en ejecutar lo que se ha programado en la primera fase del ciclo Shewhart/Deming citado por Deming (1989).
Desde esta perspectiva los resultados indican que en las empresas objeto de estudio, la fase de implementación, es decir la ejecución de las actividades planificadas, no se llevan a cabo en su totalidad, puesto que se realizan a veces, ocasionando que los objetivos y metas propuestas no se alcancen completamente.

\section{Verificación}

En cuanto al proceso de gestión ambiental, en el gráfico 3 se observa que el indicador verificación refleja como resultado un promedio de 2,4. Por lo tanto, en el baremo de respuesta se ubica en la categoría baja presencia. Los resultados demuestran que la empresa con mayor promedio obtenido es POLINTER con un índice de 2,5; seguido de PRALCA, cuya media es 2,4 ; y por último se encuentra PROPILVEN con un promedio de 2,3. Según las respuestas emitidas por los encuestados, las empresas en cuestión se ubican en la categoría relativa a baja presencia, lo cual indica que escasamente se están verificando las actividades planificadas.

Partiendo de los planteamientos señalados por Granero y Ferrando (2007), las empresas objeto de estudio casi nunca realizan el seguimiento ni la medición de sus procesos. Sin embargo, los resultados permiten establecer que solamente se realiza la evaluación de los requisitos legales aplicables en materia ambiental, obviando elementos importantes relacionados con el control de los registros, las acciones preventivas, las acciones correctivas y las auditorías.

Desde esta perspectiva, según lo establecido en la norma 14001 (2015), en cuanto a la vinculación del indicador 
verificación con la tercera fase del ciclo Shewhart/Deming citada por Deming (1989) y denominada verificar, los resultados obtenidos permiten inferir que en las empresas objeto de estudio no se está realizando una evaluación adecuada en cuanto a la gestión de los aspectos ambientales, los cuales involucran la verificación de una serie de procedimientos asociados al control de las actividades que pueden tener un impacto negativo en el ambiente.

Cabe destacar que los resultados obtenidos concuerdan con los planteamientos señalados por Almeida (2010), debido a que las empresas objeto de estudio carecen de la documentación requerida para la verificación de sus actividades. Lo anteriormente expuesto, permite afirmar que en las empresas estudiadas no se verifica el cumplimiento de los objetivos y metas ambientales, por ello resulta difícil evaluar, controlar y mejorar el desempeño ambiental existente en las empresas mixtas del Complejo Petroquímico Ana María Campos del estado Zulia.

\section{Revisión por la dirección}

Finalmente, los resultados del indicador referido a la revisión por la dirección se muestran en el gráfico 3, los cuales corresponden a un valor de 2,2 como promedio, ubicando al indicador en el baremo de respuestas referido a la categoría baja presencia. Se puede observar que la empresa con mayor promedio obtenido es POLINTER, con un índice de 2,4; seguida de PROPILVEN y PRALCA con un promedio de 2,2. Los promedios obtenidos permiten ubicar a las empresas en la categoría baja presencia, según el baremo de interpretación.
De acuerdo a los resultados obtenidos en las empresas objeto de estudio, la alta dirección casi nunca realiza la evaluación del desempeño ambiental, por lo tanto, no puede valorar la adecuación ni la eficacia de la gestión ambiental en las empresas en cuestión.

Así mismo, las respuestas emitidas por los sujetos encuestados permiten afirmar que las empresas estudiadas no realizan las evaluaciones pertinentes, lo cual dificulta la toma de decisiones para la mejora continua de la gestión ambiental, tal y como lo expresan Granero y Ferrando (2007), es decir, que la alta dirección no formula recomendaciones para mejorar el desempeño ambiental en las empresas mixtas del Complejo Petroquímico Ana María Campos.

Dentro de este marco, según lo establecido en la norma ISO 14001 (2015) en cuanto a la vinculación del indicador revisión por la dirección con la fase actuar del ciclo Shewhart/Deming citado por Deming (1989), los resultados obtenidos hacen suponer que en las empresas objeto de estudio no se revisan ni se emprenden las acciones necesarias para mejorar los aspectos débiles encontrados en las fases anteriores.

Desde una perspectiva más general, el promedio obtenido en la dimensión correspondiente al proceso de gestión ambiental $(2,5)$ se ubica, según el baremo de interpretación, en la categoría concerniente a baja presencia. Adicionalmente, en el gráfico 4 se muestra el comportamiento de esta dimensión en las empresas estudiadas.

En función de los resultados presentados en el gráfico 4 se puede afirmar que en las empresas objeto de estudio no se lleva a cabo el proceso de gestión ambiental, puesto 
que el promedio de la dimensión demuestra que casi nunca se cumplen las actividades inherentes a las cuatro fases planteadas por Deming (1989), basadas en planificar, hacer, verificar y actuar.

Desde esta perspectiva, los resultados obtenidos permiten inferir que las empresas objeto de estudio carecen del compromiso necesario para lograr un proceso de gestión ambiental eficaz y eficiente, puesto que las mismas solo cumplen con la regulación ambiental vigente dejando a un lado el objetivo principal de la gestión ambiental, el cual está referido a la emisión de cero contaminantes al ambiente. Así mismo, no adoptan las estrategias necesarias que impulsen la protección ambiental a través del cumplimiento sistemático de las cuatro fases definidas en el ciclo Shewhart/Deming.
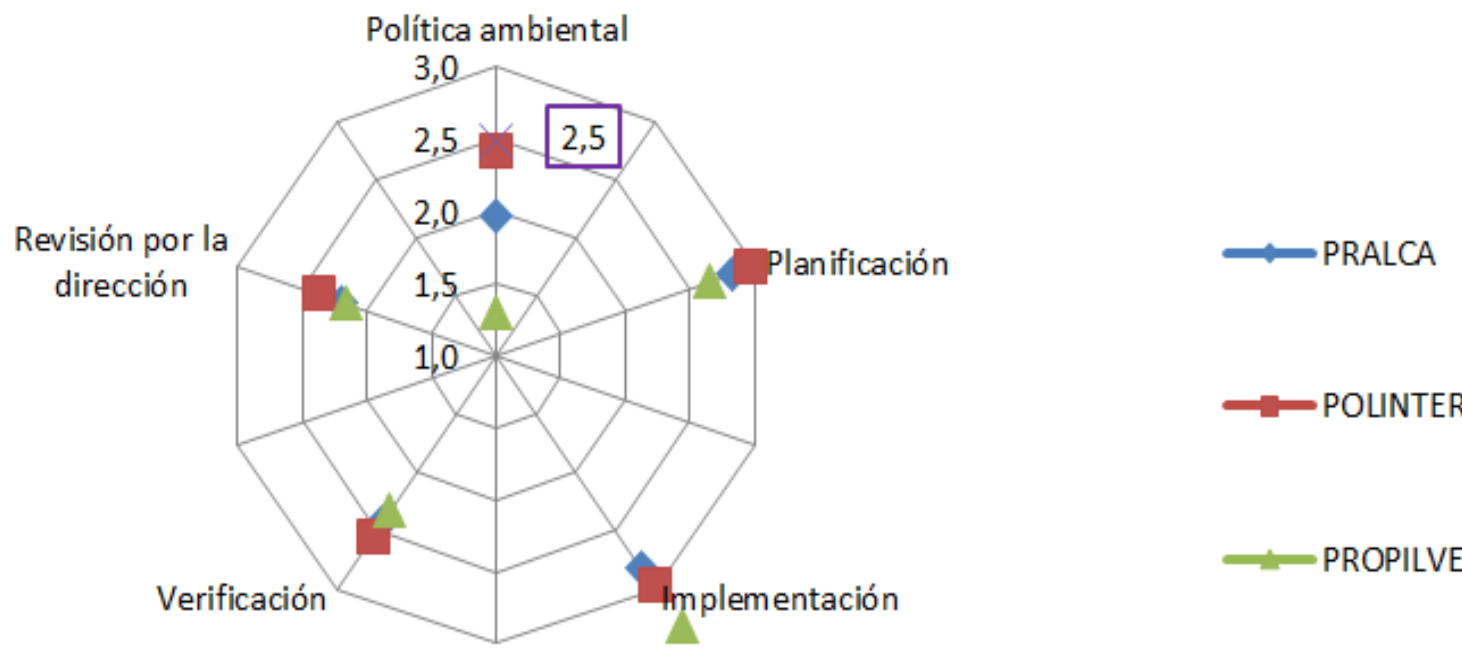

PROPILVEN

PROMEDIO

DIMENSIÓN

Figura 4. Comportamiento de la dimensión proceso de gestión ambiental en las empresas mixtas del complejo Petroquímico Ana María Campos.

En este sentido, los resultados obtenidos en la presente investigación difieren de los planteamientos de Alperstedt et al. (2010), puesto que en las empresas objeto de estudio la alta dirección no muestra el compromiso necesario para emprender un sistema de gestión ambiental acorde con los requisitos establecidos en la norma ISO 14001 (2015).
Los planteamientos anteriores hacen suponer que las empresas encuestadas presentan debilidades para completar las fases del ciclo Shewhart/Deming citado por Deming (1989), debido a que la alta dirección representada en cada una de las empresas objeto de estudio, requieren estrategias que permitan impulsar el establecimiento y la implementación de un 
sistema de gestión ambiental que se adapte a sus necesidades. Esto coincide con lo expuesto por Acuña et al. (2017:150) al afirmar que "las empresas certificadas con el sistema de gestión ambiental ISO 14001-04 presentan un aprovechamiento apenas aceptable de los beneficios y ventajas competitivas que genera esta norma".

\section{CONCLUSIONES}

En cuanto a los aspectos ambientales, las empresas objeto de estudio siempre cumplen con la regulación ambiental vigente en cuanto a la ejecución de los estudios ambientales referidos a la caracterización de efluentes, estudio de calidad de aire y estudio de ruido ambiental, pero no cuentan con procedimientos documentados, ni metodologías que les permitan identificar y valorar los aspectos ambientales derivados de sus actividades, lo cual dificulta la implementación de medidas para mitigarlos.

Las empresas estudiadas cuentan con controles para el monitoreo de los aspectos ambientales existentes en las empresas objeto de estudio como: la evaluación rutinaria del agua, los análisis de laboratorio, tanto internos como externos, identificación, cuantificación, almacenamiento y disposición final de desechos peligrosos a través de terceros, y un sistema de inyección de vapor al quemador (mechurrio) para controlar las emisiones atmosféricas. Adicionalmente, se requiere realizar un procedimiento que permita a través de una metodología certificada identificar otros aspectos ambientales que se encuentran intrínsecamente relacionados con sus actividades como lo son, el uso de energía, la optimización de materia primas y el uso del espacio, entre otros.

Desde otra perspectiva, en cuanto al proceso de gestión ambiental en las empresas mixtas del complejo petroquímica Ana María Campos del estado Zulia, se pudo evidenciar a través de los resultados obtenidos que el proceso de gestión casi nunca se lleva a cabo, puesto que no se encuentran identificadas en su totalidad las cuatro fases del ciclo Shewarth/Deming vinculadas a planificar, hacer, verificar $y$ actuar. Así mismo, es necesario iniciar un plan de trabajo para la elaboración e implementación de un sistema de gestión ambiental.

\section{REFERENCIAS}

Acuña, N., Figueroa, L. y Wilches, M. (2017). Influencia de los Sistemas de Gestión Ambiental ISO 14001 en las organizaciones: caso estudio empresas manufactureras de Barranquilla. Ingeniare. Revista chilena de ingeniería 25(1), 143-153. DOI: 10.4067/S071833052017000100143

Almeida, E. (2010). Elaboración de un Sistema de Gestión Ambiental para el Taller de Facilidades de Superficie de la gerencia de Exploración y Producción en Lago Agrio. Tesis de Grado previa a la obtención del Título de Magíster en Gestión Ambiental y la Industria. Universidad Internacional SEK. QuitoEcuador

Alperstedt, G., Quintella, R. y Souza, L. (2010). Estrategias de gestión ambiental y sus factores determinantes: Un análisis Institucional. Revista RAE 50(18), 170$186 . \quad$ DOI: $10.1590 /$ S003475902010000200004

Álvarez, F. (2013). Calidad y Auditoria en Salud. $2^{\text {da }}$ edición. Editorial ECOE. Bogotá.

Castells, X. (2012). Clasificación y gestión de residuos. Reciclaje de residuos industriales. Editorial Díaz de Santos. Madrid

Cuadros, J. y Téllez, J. (2019). Sistema Único Integrado de Gestión: calidad, ambiente, seguridad y salud. Teuken Bidikay 10(14), 
121-156. DOI: 10.33571/teuken.v10n $14 a 6$

Decreto 2217 (1992). Normas sobre control de la contaminación generada por ruido. Gaceta Oficial № 4.418 de fecha 27 de abril de 1992. Caracas-Venezuela

Deming, W. (1989). Calidad, productividad y competitividad. La salida de la crisis. Ediciones Díaz de Santos. Madrid

González, C. y Morales, J. (2010). Apropiación de un modelo de gestión ambiental en Grupo Bimbo y Barser. Memorias $V$ Congreso Internacional de Sistemas de Innovación para la Competitividad. Tecnologías Convergentes para la Competitividad. MT9 Innovación y Ecología industrial. ISBN: 978-607-95030-7-9. XochimilcoMéxico

Granero, J. y Ferrando, M. (2007). Como implantar un sistema de gestión ambiental según la norma ISO 14001:2004. $2^{\text {da }}$ edición. Editorial FC. España

Hernández, Y. (2012). Gestión ambiental como estrategia para la competitividad de las universidades privadas. Trabajo de grado para optar al Título de Doctora en Ciencias Gerenciales. Universidad Dr. Rafael Belloso Chacín, Decanato de Investigación y Postgrado, Doctorado en Ciencias Gerenciales, Maracaibo

Hurtado, J. (2010). Metodología de la investigación: guía para una comprensión holística de la ciencia. $4^{\text {ta }}$ edición. Editorial Quirón. Caracas

Infante, H. y Robles, M. (2008). Sistema de gestión ambiental en las empresas productoras de resinas plásticas. ¿̇Se cumplen los requisitos? Revista de Estudios Interdisciplinarios en Ciencias Sociales 10(2), 277-289

Jiménez, S. y Ortega, I. (2011). Análisis de la vinculación interorganizacional gobiernoindustria del plástico para la producción de innovaciones en la gestión ambiental. Memorias del VI Congreso Internacional de Sistemas de Innovación para la Competitividad. Agentes de innovación: hacia una economía sostenible en I+D+I. MT5 Ecología Industrial e Innovación. Ciudad de León Guanajuato-México

Ley Orgánica del Ambiente (2006). Gaceta Oficial Extraordinaria № 5.833 de fecha 22 de diciembre de 2006. CaracasVenezuela

Matilla, K. (2008). Los modelos de la planificación estratégica en la teoría de las relaciones públicas. $1^{\text {era }}$ edición. Editorial UOC. Barcelona

Norma Internacional ISO 14001 (2015). Sistema de gestión ambiental-Requisitos con orientación para su uso. Norma internacional. Traducción certificada

Pousa, X. (2006). ISO 14001: un sistema de gestión ambiental. 1era edición. Editorial Ideas Propias. Vigo 